\title{
Inability to reduce morbidity of diarrhea by ORS: can we design a better therapy?
}

\author{
Jane E. Harrell ${ }^{1}$ and Sam X. Cheng ${ }^{2}$
}

Diarrheal disease is a worldwide problem that still causes significant morbidity and mortality among children. Currently, oral rehydration solution (ORS) is the standard of care for acute diarrhea in pediatric patients. Although effective in reducing mortality, ORS does not alleviate diarrheal symptoms, thus reducing caregiver compliance and therapeutic efficacy. This article will briefly review the current problem of pediatric diarrhea and the shortcomings of current therapies; however, the focus of this review is to examine the intestinal calcium-sensing receptor (CaSR). The author summarizes the evidence suggesting that targeting the CaSR will enable clinicians to address all four major pathophysiological mechanisms of diarrheal disease, and substantiates the need for future research regarding this therapy.

\section{HISTORY OF ORAL REHYDRATION SOLUTION USE IN DIARRHEA}

D espite a decrease in worldwide diarrhea-related mortality over the past 30 years, diarrheal disease remains the fourth leading cause of death in children under the age of 5, with 499,000 dying in 2015. Whereas mortality has decreased to $39.2 \%$ from 2005 to 2015 , morbidity has not declined to a comparable degree. In 2015, there were 2.39 billion estimated cases of diarrhea. Of these cases, 957.5 million occurred in children under the age of 5 ((ref. 1)). In the United States from 2001 to 2006, the cost of hospitalizations, emergency room visits, and outpatient visits for diarrhea in pediatric patients was $\sim \$ 10$ billion per year, not including indirect costs for the family. Notably, 77\% of children visit an outpatient clinic for diarrhea by the age of 5 ( (ref. 2)). The most common cause of acute diarrhea is infectious gastroenteritis; however, the majority of diarrhearelated deaths are the result of dehydration and electrolyte imbalance, and not infection (3).

The development of the current oral rehydration therapy dates back to over 50-years ago. In 1958, Crane and his colleagues discovered that intestinal $\mathrm{Na}^{+}$and glucose absorption are coupled. The researchers also learned that $\mathrm{Na}^{+} /$glucose cotransport plays a significant role in the reabsorption of water (4). Understanding this mechanism proved vital in developing a treatment for Cholera. Although Cholera toxin effectively inhibits many intestinal electrolyte transporters, $\mathrm{Na}^{+} /$glucose co-transport remains undisturbed. In 1968, researchers working in Bangladesh and India exploited this principle and formulated a mixture of glucose, sodium, potassium, and chloride, now called oral rehydration solution (ORS) (5).

Cholera causes secretory diarrhea; the toxin increases fluid secretion and decreases fluid absorption in the intestine. The alpha-subunit of the Cholera toxin constitutively activates adenylyl cyclase, increasing the amount of cyclic adenosine monophosphate (cAMP) in the cell, which in turn stimulates protein kinase A. When activated, protein kinase A phosphorylates the cystic fibrosis transmembrane conductance regulator (CFTR) channel, causing secretion of $\mathrm{Cl}^{-}$and water. In addition, high levels of cAMP inhibit $\mathrm{NaCl}$ absorption, $\mathrm{Na}^{+} / \mathrm{H}^{+}$exchange, and $\mathrm{Cl}^{-} / \mathrm{HCO}_{3}{ }^{-}$exchange, resulting in decreased electrolyte and water absorption $(6,7)$. $\mathrm{Na}^{+}$/glucose co-transport is cAMP-independent; therefore, solute and water can be reabsorbed despite the presence of high cAMP levels. Certain strains of Escherichia coli and other bacteria produce enterotoxins that cause secretory diarrhea in a similar manner. Consequently, clinicians have expanded the use of ORS to treat diarrhea-related dehydration caused by pathogens other than Cholera, including viruses.

The pathophysiology of rotavirus gastroenteritis can be partially attributed to a viral-encoded enterotoxin NSP4, which also results in excessive $\mathrm{Cl}^{-}$secretion. However, this secretion is distinct from increased cAMP-dependent CFTR activity, and the identity of the $\mathrm{Cl}^{-}$channel remains unknown. Importantly, NSP4 also impairs intestinal cells through the inhibition of $\mathrm{Na}^{+}$/glucose co-transport, the main mechanism by which ORS enhances absorption (8). As a result, ORS therapy may not be as effective in patients infected by rotavirus because of this inhibition.

Currently, ORS is the only oral therapy available for acute diarrhea in children. The use of ORS has significantly decreased mortality from diarrheal disease; however, its usage has not increased but decreased since 2000, with fewer than $33 \%$ of children with diarrhea under the age of 5 using the therapy (9). Often, caregivers are reluctant to use ORS because it takes a relatively long time to produce positive

${ }^{1}$ College of Medicine, University of Florida, Gainesville, Florida; ${ }^{2}$ Department of Pediatrics, Gastroenterology, Hepatology, and Nutrition, University of Florida, Gainesville, Florida. Correspondence: Sam X. Cheng (sam.cheng@ufl.edu)

Received 12 July 2017; accepted 14 November 2017; advance online publication 3 January 2018. doi:10.1038/pr.2017.295 


\section{Review | Harrell and Cheng}

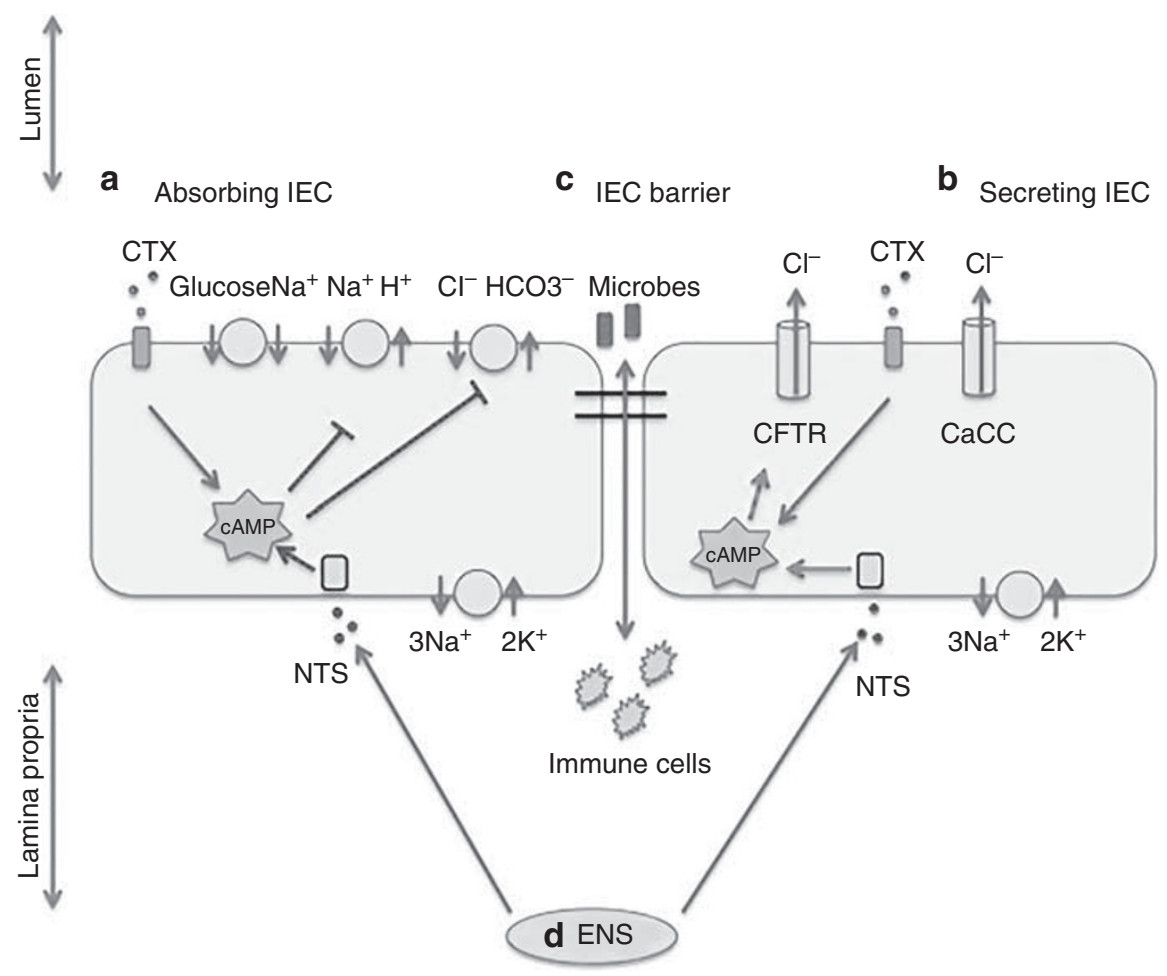

Figure 1. Four pathways leading to the formation of diarrhea. (a) Impaired absorption, (b) excessive secretion, (c) intestinal barrier breakdown and enhanced inflammation, (d) overly active enteric nervous system. CaSR activators reduce changes in all the four diarrhea-causing pathways. CaCC, calcium-activated chloride channel; CFTR, cystic fibrosis transmembrane conductance regulator; CTX, cholera toxin; ENS, enteric nervous system; IEC, intestinal epithelial cell; NTS, neurotransmitters.

results. In addition, despite attempts to modify it, including lowering osmolality to reduce the risk of increased diarrhea volume, ORS does nothing to prevent excessive secretion of fluid, the major mechanism by which Cholera and other infectious organisms cause diarrhea. In fact, the glucose contained in ORS stimulates calcium-activated chloride secretion in the small intestine, transiently increasing diarrhea volume (10). Again, this translates to decreased levels of caregiver compliance and to the misconception that ORS is not an effective therapy.

\section{CURRENT ANTIDIARRHEAL THERAPIES}

There are four categories of antidiarrheal treatment: proabsorptive, antisecretory, antimotility, and anti-inflammatory (Figure 1). Clinical management of acute diarrhea in pediatric patients is purely pro-absorptive and involves replacement of fluid with ORS or parenteral rehydrating solution (3). Currently, there are no approved therapies in the United States that stop fluid loss or decrease gut motility, which are efficacious and safe to use in children. Several experimental therapies have been considered. Loperamide, an antimotility drug that has been used with success in adults, can cause serious side effects such as ileus, lethargy, and death in children (11). A promising CFTR and calcium-activated chloride channel inhibitor, Crofelemer, proved to be a weak and partial antagonist of CFTR (12), limiting its potential positive effects against diarrhea caused by excessive CFTR activity.

Although ORS decreases mortality from dehydration and electrolyte imbalance, it does not alleviate symptoms. ORS does not target cAMP/cGMP accumulation or the influence of the enterotoxin NSP4, the major mechanisms causing secretory diarrhea by numerous bacteria and rotavirus, respectively. Patients continue to experience diarrhea in spite of treatment. As a result, many clinicians and caregivers turn to antimicrobial agents (7). Although antibiotics are useful in some cases of acute bacterial diarrhea, they must be used cautiously in order to prevent pathogen resistance and opportunistic infection. Certain enteric organisms such as Salmonella should not be treated with antibiotics, as they can prolong the duration of illness (13). Most importantly, antibiotics do not immediately prevent fluid and electrolyte loss-the most common cause of diarrhea-related mortality.

Although ORS is an effective lifeline for those experiencing acute diarrhea, it is not curative. Even while consuming ORS, patients experience severe symptoms and can suffer recurrent episodes of diarrhea. Children with frequent diarrheal disease are more likely to be malnourished and have impaired growth and cognitive development (14). Malnutrition is known to increase the incidence and duration of diarrhea, thus creating a viscous cycle (15). Unfortunately, there are no current therapies that address this issue in a cost-effective manner. 


\section{CaSR target to treat pediatric diarrhea ReView}

A pediatric therapy that both lessens diarrheal excretion and rehydrates is urgently needed.

\section{A PROMISING NEW TARGET FOR THERAPY}

A promising target for such a therapy is the extracellular calcium-sensing receptor (CaSR). CaSR is a G proteincoupled receptor that is expressed in various human tissues including the small and large intestine. This receptor primarily functions to maintain calcium homeostasis and to detect luminal nutrients including the following: calcium, polyamines, L-amino acids, and oligopeptides (16). Stimulation by such a variety of agonists can result in multiple distinct active conformations of the receptor, allowing for different biological effects to take place in the cell (17).

CaSR has been studied in mammals, amphibians, birds, and marine species. In the intestine of marine organisms, CaSR has been found to prevent water loss to hyperosmotic seawater surroundings (18). This function is conserved in the human intestine and is the basis for the development of CaSR-targeted antidiarrheal therapy (7). Inside the intestine, CaSR has been located in the secreting crypt epithelium, absorbing surface epithelium, motility-modulating enteric nervous tissue, and inflammatory cells (19-23,,,). Researchers investigating the role of CaSR in these cells have discovered that activation of the receptor decreases cellular cyclic nucleotides and reverses all four pathological changes occurring in diarrhea: excessive secretion, malabsorption, increased gut motility, and a heightened inflammatory response.

\section{UNDERSTANDING THE CaSR}

The two major mechanisms of secretory diarrhea, increased secretion and decreased absorption of electrolytes and fluid, are both altered by CaSR activation $(16,22-26,,,)^{\prime}$. Studies using rat colonic epithelium show that CaSR activation counteracts the effects of Cholera toxin, Escherichia coli heatstable enterotoxin STa and other secretagogues by inhibiting cyclic nucleotide accumulation and anion secretion (24). By stimulating the enzyme phosphodiesterase, CaSR leads to the degradation of cyclic nucleotides. Decreased cAMP/cGMP concentrations reduce CFTR translocation to the apical membrane and enhance both $\mathrm{Na}^{+} / \mathrm{H}^{+}$and $\mathrm{Cl}^{-} / \mathrm{HCO}_{3}{ }^{-}$ exchange. Thus, CaSR activation simultaneously normalizes fluid excretion and reabsorption.

Cyclic nucleotides also have a critical role in regulating the enteric nervous system (ENS), which controls $~ 50 \%$ of fluid secretion in infectious diarrhea (27). When stimulated by cAMP, endocrine intestinal cells secrete neurotransmitters, such as serotonin and neurotensin. These peptides are activators of the ENS and initiate an enteric neural response through the stimulation of submucosal plexus neurons, which ultimately augment secretion and inhibit absorption (6). Similarly, the ENS is activated in rotavirus-induced diarrhea. It has been postulated that NSP4 may bind to enterochromaffin cells, prompting the release of serotonin, leading to stimulation of the ENS and fluid loss (8).
When the mammalian small intestine is treated with Cholera toxin, fluid secretion is reduced by two neurotoxins: tetrodotoxin (TTX) and hexamethonium (6). TTX blocks voltage-gated $\mathrm{Na}^{+}$channels, whereas hexamethonium prevents acetylcholine from binding to nicotinic receptors; both effectively suppress the ENS. A study by Cheng et al. examined the effect of CaSR on ENS-mediated fluid secretion by analyzing short-circuit current $\left(I_{\mathrm{sc}}\right)$ through rat colons. The group applied secretagogues to the epithelium with and without TTX. $I_{\mathrm{sc}}$ increased to a greater extent in the absence of TTX, indicating that fluid secretion is greater without ENS inhibition. Later, CaSR was activated using R568, a calcimimetic. Interestingly, CaSR activation decreased $I_{\mathrm{sc}}$ in tissue that was not treated with TTX. When TTX was administered, R568 did not significantly reduce $I_{\mathrm{sc}}$, suggesting that the stimulated CaSR reduces fluid secretion by manipulating ENS activity (22).

Finally, a study examining CaSR knockout mice showed that CaSR activation promotes an anti-inflammatory response in the intestine that could prove to be beneficial in diarrhea caused by enterocolitis and inflammatory bowel disease. CaSR knockout mice have decreased expression of tight junction molecules, such as claudin 2,8 , and 14, which help form a strong intestinal barrier. In addition, their intestinal barrier function is compromised, as seen by significantly reduced transepithelial electrical resistance and increased flux to fluorescein isothiocyanate-conjugated dextran (28). This leaky barrier allows microbes to easily traverse from the intestinal lumen through the paracellular space into the lamina propria, activating an inflammatory response. Mice without CaSR intestinal expression also have reduced secretion of antimicrobial peptides that protect against bacterial translocation from the lumen into the blood. A gene array analysis of CaSR-1- mouse colonic epithelium showed an increase in the expression of several proinflammatory cytokine-encoding genes, including interleukin (IL)- $1 \beta$, tumor necrosis factor- $\alpha$, and IL-6, further supporting the role of CaSR in intestinal immune homeostasis (28).

\section{AN IMPROVED ORS}

CaSR is a very encouraging target for diarrheal disease, as activation can reduce symptoms caused by all four pathophysiological mechanisms, although other drugs target only one. Fortunately, CaSR agonists are easily accessible, inexpensive, and safe to use in the pediatric population. The primary ligand for CaSR is calcium, an essential nutrient that is commonly found and fortified in foods. Allosteric activators of CaSR include the polyamine spermine that is found in breast milk and the amino-acid tryptophan. CaSR adopts multiple active conformations, as it is constantly exposed to these agonists in the intestine. Adding nutrients to ORS would be simple and could possibly increase the efficacy of the current therapy. Small additions of spermine can markedly enhance activation of CaSR, even when calcium is at a physiologically normal level (25). Secondarily, polyamines and amino acids would provide essential nutrition to 
malnourished children suffering from diarrhea. Different agonist combinations should be investigated to identify which combinations and doses are the most therapeutic with the fewest adverse effects.

As expected, studies have shown that these nutrients are safe to use. Intake of oral calcium slightly above the recommended daily allowances has shown positive effects, reducing diarrhea in preliminary clinical trials $(29,30)$. It is probable that CaSR activation had a role in reducing diarrhea volume and accelerated the recovery period in these patients. A transient increase in calcium intake did not alter bloodcalcium homeostasis. The patients' urinary and blood calcium levels remained normal, and the only noticeable side effect was mild constipation that subsided with completion of treatment. Calcium absorption from the intestine is a process that is heavily influenced by the presence of vitamin D. Without additional vitamin $\mathrm{D}$, the majority of extra calcium remains in the intestine, locally stimulating CaSR without systemic effects.

Although these results are promising, the clinical trials were completed in very specific patient populations and cannot be generalized. Some of the patients included were hypocalcemic to begin with; therefore, increasing their calcium intake slightly above the recommended daily allowance is unlikely to have a negative effect. It is probable that a calcium concentration above the recommended daily allowance would be necessary to produce a sufficiently potent therapy even with the addition of amino acids and polyamines, creating a potential safety concern. Randomized controlled trials are needed to establish a concentration of calcium that is both effective and safe. Calcimimetic drugs, such as the chemical compound R568, are more potent and could have therapeutic effects at smaller concentrations (24). Nevertheless, calcium is a more attractive remedy because it is widely available and inexpensive. A new formulation of ORS with calcium and other nutrient CaSR agonists could more easily be implemented as standard treatment around the world.

\section{CONCLUSION}

The CaSR is a promising target for pediatric antidiarrheal therapy. In contrast to conventional ORS, CaSR-based treatment is a four-pronged approach, addressing all major pathophysiological mechanisms of diarrhea. Initial clinical studies have produced positive results, demonstrating that a CaSR-ORS would be safe, affordable, and possibly more effective than the current gold standard. By relieving the symptoms associated with diarrheal disease, CaSR-ORS could inspire more confidence in caregivers, increasing overall compliance and reducing mortality worldwide.

\section{ACKNOWLEDGMENTS}

We would like to extend our gratitude to Johnathan J. Fraebal at the University of Florida for discussing and reviewing the manuscript.

\section{STATEMENT OF FINANCIAL SUPPORT}

This work was supported in part by Eunice Kennedy Shriver National Institute of Child Health and Human Development of the National
Institutes of Health HD079674 (S.X.C.) and HD092133 (S.X.C.), University of Florida funds (S.X.C.), and Children's Miracle Network (S.X.C.).

Disclosure: The authors declare no conflict of interest.

\section{REFERENCES}

1. Troeger C, Forouzanfar M, Rao PC, et al. Estimates of global, regional, and national morbidity, mortality, and aetiologies of diarrhoeal diseases: a systematic analysis for the Global Burden of Disease Study 2015. Lancet Infect Dis 2017;3099:1-40.

2. Cortes JE, Curns AT, Tate JE, et al. Trends in healthcare utilization for diarrhea and rotavirus disease in privately insured US children $<5$ years of age, 2001-2006. Pediatr Infect Dis J 2009;28:874-8.

3. Fleisher GR. Approach to diarrhea in children in resource-rich countries. In: Post TW, ed. UpToDate. Waltham, MA, 2017. (https://www.uptodate. com/contents/approach-to-diarrhea-in-children-in-resource-rich-countries? source $=$ search_result\&search $=$ acute diarrhea pediatrics\&selectedTitle $=2$ $\sim 150$ ). Accessed 8 June 2017.

4. Hamilton KL, Robert K. Crane-Na+-glucose cotransporter to cure? Front Physiol.2013;4:53.

5. Guerrant RL, Carneiro-Filho BA, Dillingham RA. Cholera, diarrhea, and oral rehydration therapy: triumph and indictment. Clin Infect Dis 2003;37:398-405.

6. Field M. Intestinal ion transport and the pathophysiology of diarrhea. J Clin Invest 2003;111:931-43.

7. Cheng SX. Calcium-sensing receptor: a new target for therapy of diarrhea. World J Gastroenterol 2016;22:2711-4.

8. Ramig RF. Pathogenesis of intestinal and systemic rotavirus infection. J Virol 2004;78:10213-20.

9. Donowitz M, Alpers DH, Binder HJ, et al. Translational approaches for pharmacotherapy development for acute diarrhea. Gastroenterology 2012;142:e1-9.

10. Yin L, Vijaygopal P, MacGregor GG, et al. Glucose stimulates calciumactivated chloride secretion in small intestinal cells. Am J Physiol 2014;306:C687-96.

11. Li S-TT, Grossman DC, Cummings P. Loperamide therapy for acute diarrhea in children: systematic review and meta-analysis. PLoS Med 2007;4:e98.

12. Tradtrantip L, Namkung W, Verkman AS. Crofelemer an antisecretory antidiarrheal proanthocyanidin oligomer extracted from Croton lechleri, targets two distinct intestinal chloride channels. Mol Pharmacol 2010;77: 69-78.

13. Onwuezobe IA, Oshun PO, Odigwe CC. Antimicrobials for treating symptomatic non-typhoidal Salmonella infection. Cochrane Database Syst Rev 2012;11:CD001167.

14. Petri WA, Miller M, Binder HJ, et al. Enteric infections, diarrhea, and their impact on function and development. J Clin Invest 2008;118: 1277-90.

15. Guerrant RL, Schorling JB, McAuliffe JF, et al. Diarrhea as a cause and an effect of malnutrition: diarrhea prevents catch-up growth and malnutrition increases diarrhea frequency and duration. Am J Trop Med Hyg 1992;47:28-35.

16. Tang L, Cheng CY, Sun X, et al. The extracellular calcium-sensing receptor in the intestine: evidence for regulation of colonic absorption, secretion, motility, and immunity. Front Physiol 2016;7:245.

17. Thomsen ARB, Hvidtfeldt M, Brauner-Osborne H. Biased agonism of the calcium-sensing receptor. Cell Calcium 2012;51:107-6.

18. Nearing J, Betka M, Quinn S, et al. Polyvalent cation receptor proteins (CaRs) are salinity sensors in fish. Proc Natl Acad Sci USA 2002;99: 9231-6.

19. Kelly JC, Lungchukiet P, John MacLeod R. Extracellular calcium-sensing receptor inhibition of intestinal epithelial TNF signaling requires CaSRmediated Wnt5a/Ror2 interaction. Front Physiol 2011;2:17.

20. Yamaguchi T, Olozak I, Chattopadhyay N, et al. Expression of extracellular calcium $(\mathrm{Ca} 2+\mathrm{o})$-sensing receptor in human peripheral blood monocytes. Biochem Biophys Res Commun 1998;246:501-6. 
21. Chattopadhyay N, Cheng I, Rogers K, et al. Identification and localization of extracellular Ca2+-sensing receptor in rat intestine. Am J Physiol 1998;274:G122-30.

22. Cheng SX. Calcium-sensing receptor inhibits secretagogue-induced electrolyte secretion by intestine via the enteric nervous system. Am J Physiol 2012;303:G60-70.

23. Cheng SX, Okuda M, Hall AE, et al. Expression of calcium-sensing receptor in rat colonic epithelium: evidence for modulation of fluid secretion. Am J Physiol 2002;283:G240-50.

24. Geibel J, Sritharan K, Geibel R, et al. Calcium-sensing receptor abrogates secretagogue- induced increases in intestinal net fluid secretion by enhancing cyclic nucleotide destruction. Proc Natl Acad Sci USA 2006;103:9390-7.

25. Cheng SX, Geibel JP, Hebert SC. Extracellular polyamines regulate fluid secretion in rat colonic crypts via the extracellular calcium-sensing receptor. Gastroenterology 2004;126:148-58.
26. Tang L, Peng M, Liu L, et al. Calcium-sensing receptor stimulates Cl- and SCFA-dependent but inhibits cAMP-dependent HCO3- secretion in colon. Am J Physiol 2015;308:G874-83.

27. Lundgren $O$. Role of the enteric nervous system in the fluid and electrolyte secretion of rotavirus diarrhea. Science 2000;287:491-5.

28. Cheng SX, Lightfoot YL, Yang T, et al. Epithelial CaSR deficiency alters intestinal integrity and promotes proinflammatory immune responses. FEBS Lett 2014: 4158-66.

29. Bovee-Oudenhoven IMJ, Lettink-Wissink MLG, Van Doesburg W, et al. Diarrhea caused by enterotoxigenic Escherichia coli infection of humans is inhibited by dietary calcium. Gastroenterology 2003;125: 469-76.

30. Cheng SX, Bai HX, Gonzalez-Peralta R, et al. Calcium ameliorates diarrhea in immunocompromised children. J Pediatr Gastroenterol Nutr 2013;56:641-4. 